\title{
On a functional equation related to projections of abelian groups
}

JÜRG R̈̈TZ

Dedicated in friendship to Professor János Aczél on his eightieth birthday

Summary. For an abelian group $(G,+, 0)$ we consider the functional equation

$$
f: G \longrightarrow G, f(x+y+f(y))=f(x)+2 f(y) \quad(\forall x, y \in G),
$$

most times together with the condition $f(0)=0$. A solution of (1) is always idempotent. Our main question is as to whether it must be additive, i.e., a projection of the abelian group $G$.

Mathematics Subject Classification (2000). 39B12, 39B52.

Keywords. Abelian groups, composite functional equations, projections.

\section{Introduction, notation and preliminaries}

The subject of this paper is the composite nonlinear functional equation (1) above. We consider it here in the case of an abelian group $(G,+, 0)$ (cf. [12], [13]). The situation for a not necessarily abelian group will be considered in a forthcoming paper. The similar looking equation

$$
f: G \longrightarrow G, f(x+f(y))=f(x)+f(y) \quad(\forall x, y \in G)
$$

has been thoroughly investigated ([2], pp. 326-327; [5], p. 6.16, (14)).

There are, however, serious differences between the theories of $(1)$ and $\left(1^{\prime}\right)$, respectively, e.g., in $\left(1^{\prime}\right)$, the image $f(G)$ must be a subgroup of $G$ while this is not so in (1) (cf. Example 2.7 below). Furthermore, neither (1) nor $\left(1^{\prime}\right)$ implies the other.

Our main interest is in the question as to whether (1) implies

$$
f(x+y)=f(x)+f(y), \quad f[f(x)]=f(x) \quad(\forall x, y \in G),
$$

expressing that $f$ is an idempotent endomorphism, i.e., a projection of $G$. It is easily seen that (2) implies (1), which means that additivity and idempotency of $f$ can be compressed into the single functional equation (1). It was proposed in 
[3] to prove (1) $\Longleftrightarrow(2)$ for $G=\mathbb{R}$, which amounts to saying that (1) constitutes a characterization of the projections of the $\mathbb{Q}$-vector space $\mathbb{R}_{[\mathbb{Q}]}$ onto its $\mathbb{Q}$-linear subspaces. For abelian groups other than $\mathbb{R}$, the situation may be quite different: A solution of (1) need not be additive (cf. Example 2.7 or Lemma 4.7 a), d)).

For solutions $f$ of (1) many features of the projections of $G$ will be observed (Section 2), but here they are, to an essential extent, obtained in the possible absence of additivity of $f$. Section 3 deals with the cases of divisible, locally cyclic, torsion-free, and indecomposable abelian groups while section 4 treats that of torsion groups.

Notation. The symbol $:=$ means that the right-hand side defines the left-hand side,$=_{(\ldots)}=$ is a short form of quotation of $(\ldots)$, and $\square$ marks the end of a proof. $i_{A}$ denotes the identity mapping of the set $A$ and $\underline{a}$ the constant mapping with value $a . \mathbb{P}, \mathbb{N}, \mathbb{N}^{0}, \mathbb{Z}, \mathbb{Q}, \mathbb{R}$ stand for the sets of prime numbers, positive integers, nonnegative integers, integers, rational and real numbers, respectively.

Throughout the paper, $(G,+, 0)$ or $(G,+)$ or $G$ denotes an abelian group.

$\omega_{2}: G \longrightarrow G, \omega_{2}(x):=2 x(\forall x \in G)$, is an endomorphism of $G$, i.e., is in $\operatorname{End}(G)$. For $x \in G$, we use ord $x$ for the order of $x$, and for $M \subset G,\langle M\rangle$ is the subgroup of $G$ generated by $M ;\langle y\rangle:=\langle\{y\}\rangle(\forall y \in G) . H \hookrightarrow G$ expresses that the group $H$ is embeddable into $G$, i.e., that $H$ is isomorphic to a subgroup of $G$. We use 0 for the identity element of $G$ as well for the integer zero; it will always be clear from the context what is meant. We freely use the fact that for abelian groups $A$ and $B$ we have $A \times B \cong A \oplus B$, e.g., by denoting the elements of $A \oplus B$ as ordered pairs $(\alpha, \beta) \in A \times B$ whenever we find it convenient (cf. also Remark 1.1 below). For every $n \in \mathbb{N}$, we let $\mathbb{Z}_{n}$ stand for the cyclic group with $n$ elements, most times written as $\{0, \ldots, n-1\}$, and $\mathbb{Z}\left(p^{\infty}\right)$ is the Prüfer quasicyclic group $(\forall p \in \mathbb{P})$.

Finally we put

$$
\begin{aligned}
\left(1_{0}\right) & :=(1) \wedge f(0)=0, \\
S(G) & :=\{f: G \longrightarrow G ; f \quad \text { is a solution of }(1)\} \\
S_{0}(G) & :=\left\{f: G \longrightarrow G ; f \quad \text { is a solution of } \quad\left(1_{0}\right)\right\} .
\end{aligned}
$$

The following remarks are easily verified.

Remark 1.1. If $G$ and $H$ are abelian groups and $\varphi: G \longrightarrow H$ is an isomorphism, $f: G \longrightarrow G, g: H \longrightarrow H, g=\varphi \circ f \circ \varphi^{-1}$, then

a) $f \in S(G) \Longrightarrow g \in S(H) ; f \in S_{0}(G) \Longrightarrow g \in S_{0}(H)$.

b) $S_{0}(G) \subset \operatorname{End}(G) \Longrightarrow S_{0}(H) \subset \operatorname{End}(H)$.

Remark 1.2. If $M$ is a set and $f: M \longrightarrow M$ is an arbitrary mapping, then $f(M)=\{y \in M ; f(y)=y\}$ if and only if $f \circ f=f$.

Remark 1.3. For an abelian group $(G,+, 0)$, the following statements are equiv- 
alent:

i) $\omega_{2}: G \longrightarrow G$ is injective.

ii) $G$ has no elements of order 2 .

iii) $x \in G \Longrightarrow \operatorname{ord} x \in \mathbb{N}$ odd, or ord $x=\infty$.

Remark 1.4. Two of the most significant features of abelian groups $(G,+, 0)$ are that all $\omega_{n}: G \longrightarrow G, \omega_{n}(x):=n x(\forall x \in G, \forall n \in \mathbb{N})$ are endomorphism of $G$ and that $G$ is a unitary $\mathbb{Z}$-module in a natural way. We tacitly shall use this many times.

\section{Results for arbitrary abelian groups}

Almost throughout the paper we shall suppose $f \in S_{0}(G)$, but not in Lemmas 2.1 and 2.3 where the results also hold in the absence of $f(0)=0$.

Lemma 2.1. If $f \in S(G)$, then:
a) $f[-f(y)]=-f(y) \quad(\forall y \in G)$.
b) $z \in f(G) \Longrightarrow-z \in f(G)$.
c) $f[f(y)]=f(-y)+2 f(y) \quad(\forall y \in G)$.
d) $y \in G \Longrightarrow[f[f(y)]=f(y) \Longleftrightarrow f(-y)=-f(y)]$.
e) $f(G)=\{y \in G ; f(y)=y\}$.
f) $f \circ f=f$, i.e., $f$ is idempotent.
g) $f$ is odd.

Proof. a) For $y \in G$ arbitrary and $x:=-f(y)$ we get $f(y)=f(x+y+f(y))={ }_{(1)}=$ $f(x)+2 f(y)=f[-f(y)]+2 f(y)$, i.e., a) holds.

b) If $z \in f(G)$, there exists $y \in G$ with $z=f(y)$, so $-z=-f(y)={ }_{\mathrm{a}}=$ $f[-f(y)] \in f(G)$.

c) For $y \in G$ and $x:=-y$ we obtain $f[f(y)]={ }_{(1)}=f(-y)+2 f(y)$.

$\mathrm{d}) \Longrightarrow: f(y)=f[f(y)]=_{\mathrm{c})}=f(-y)+2 f(y)$, so $f(-y)=-f(y) . \Longleftarrow: f[f(y)]$ $={ }_{\mathrm{c})}=f(-y)+2 f(y)=f(y)$.

e) Let $z \in f(G)$ be arbitrary. By a) $f(-z)=-z$, so $f[f(-z)]=f(-z)$, and for $y:=-z$ by d) $f(-(-z))=-f(-z)$, i.e., $f(-z)=-f(z)$ and finally $-z=f(-z)=-f(z)$, so $f(z)=z$, which establishes $f(G) \subset\{y \in G ; f(y)=y\}$. The reverse inclusion is obvious, so the assertion holds.

f) follows from e) and Remark 1.2 .

g) For every $y \in G$ we have by f) $f[f(y)]=f(y)$, so by d) $f(-y)=-f(y)$.

Lemma 2.2. $S(G)=S_{0}(G)$ if and only if $\omega_{2}: G \longrightarrow G$ is injective.

Proof. If $\omega_{2}$ is not injective, then by Remark $1.3 G$ has an element $a$ of order 2 , and then $\underline{a} \in S(G) \backslash S_{0}(G)$. If $\omega_{2}$ is injective and $f \in S(G)$, then by Lemma $\left.2.1 \mathrm{~g}\right)$ $f(0)=-f(0), 2 f(0)=0, f(0)=0$, so $f \in S_{0}(G)$, and $S(G)=S_{0}(G)$ holds. 
Lemma 2.3. $f \in S(G), x, y \in G \Longrightarrow[f(x)=f(y) \Longleftrightarrow f(x-y)=f(0)]$.

Proof. $\Longrightarrow$ : Let $f(x)=f(y)$. Then $f(x-y)+2 f(y)={ }_{(1)}=f((x-y)+y+f(y))=$ $f(x+f(y))=f(x+f(x))=f(0+x+f(x))={ }_{(1)}=f(0)+2 f(x)=f(0)+2 f(y)$, so $f(x-y)=f(0)$. $\Longleftarrow$ : Let be $f(x-y)=f(0)$. Then $f(x)+2 f(0)={ }_{(1)}=f(x+0+$ $f(0))=f(x+0+f(x-y))=f(y+(x-y)+f(x-y))={ }_{(1)}=f(y)+2 f(x-y)=$ $f(y)+2 f(0)$, therefore $f(x)=f(y)$.

In the Introduction we formulated our main question in the provisional form $(1) \stackrel{?}{\Longrightarrow}(2)$. With respect to Lemma $2.1 \mathrm{f})$ the idempotency part of $(2)$ is guaranteed by (1), so a more concise form of the main question reads: Does $\left(1_{0}\right)$ imply additivity of $f$ ? or, equivalently, does $S_{0}(G) \subset \operatorname{End}(G)$ hold?

Lemma 2.4. If $f \in S_{0}(G)$ and $K_{f}:=f^{-1}(\{0\})$, we have:

a) $K_{f}$ is a subgroup of $G$, and $f$ is constant on every coset $x+K_{f}(x \in G)$ of $K_{f}$. (So $K_{f}$ is the group of periods of $f$.)

b) $z \in K_{f} \backslash\{0\}, w \in f(G) \Longrightarrow z+w \notin f(G)$.

c) The values of $f$ on distinct cosets of $K_{f}$ are distinct.

d) If $M$ is a subsemigroup of $G$ with $K_{f} \subset M$ and if $N$ is a subset of $G$, then $f(M \cap N)=f(M) \cap f(N)$.

Proof. a) $f \in S_{0}(G)$ ensures $0 \in K_{f}$. The constancy assertion follows from

$x \in G, y \in K_{f} \Longrightarrow f(x+y)=f(x+y+f(y))={ }_{(1)}=f(x)+2 f(y)=f(x)$.

If $y, z \in K_{f}$, then $f(y+z)={ }_{(3)}=f(y)={ }_{(3)}=f(0)=0$, hence $y+z \in K_{f}$. Finally, if $y \in K_{f}$, then $f(-y)={ }_{(3)}=f(-y+y)=f(0)=0$, so $-y \in K_{f}$, and $K_{f}$ is a subgroup of $G$.

b) By Lemma 2.1 e) $f(w)=w$. Assume that $z+w \in f(G)$, then again by Lemma 2.1 e) $f(z+w)=z+w$, so $\left.z+w=f(z+w)={ }_{a}\right)=f(w)=w$, a contradiction to $z \neq 0$.

c) Let be $x, y \in G, f(x)=f(y)$. By Lemma $2.3 f(x-y)=f(0)=0$, i.e., $x-y \in K_{f}$, and so $x$ and $y$ belong to the same coset of $K_{f}$ in $G$. The contrapositive version of this is our assertion.

d) $f(M \cap N) \subset f(M) \cap f(N)$ is clear. If $v \in f(M) \cap f(N)$, then there exist $x \in M, y \in N$ with $v=f(x)=f(y)$, so, as in the proof of c), $y-x \in K_{f}$, so $y-x \in M$, therefore $y=(y-x)+x \in M+M \subset M$. Since also $y \in N$, i.e., $y \in M \cap N$, and $v=f(y) \in f(M \cap N)$, which proves the reverse inclusion $f(M) \cap f(N) \subset f(M \cap N)$.

Remark 2.5. a) $K_{f}$ is called the kernel of $f$ although $f$ need not be additive (Example 2.7). Despite this fact, parts a), c), d) of Lemma 2.4 perfectly imitate the behavior of a group homomorphism while part b) need not hold there.

b) For $f \in S_{0}(G)$, Lemma 2.4 a) opens the possibility of defining $\bar{f}: G / K_{f} \rightarrow G$, $\bar{f}\left(x+K_{f}\right):=f(x)(\forall x \in G)$ and considering $\bar{f}$ instead of $f$ in the sequel. For 
the situation of the functional equation $\left(1_{0}\right)$ this does not seem to be profitable for several reasons: The properties of $f$ heavily depend on those of the image set $f(G)$, and clearly $\bar{f}\left(G / K_{f}\right)=f(G) ; G / K_{f}$ depends on $f$, which would "individualize" the framework of the equation; for returning to $f$, a lifting of $\bar{f}$ would be unavoidable. So we prefer not to pursue this way. For a related situation cf. Remark 2.10 below.

Lemma 2.6. For $f \in S_{0}(G)$ and $K_{f}:=f^{-1}(\{0\})$, we have:

a) $f(x-f(x))=0(\forall x \in G)$.

b) $K_{f} \cap f(G)=\{0\}$, and every $x \in G$ has a unique representation $x=x_{1}+x_{2}$ with $x_{1} \in K_{f}, x_{2} \in f(G)$. We shall abbreviate this fact from now on by $G=K_{f} \oplus f(G)$, even in the case that $f(G)$ is not a subgroup of $G$.

c) $f(x) \in x+K_{f}=K_{f}+x(\forall x \in G)$.

Proof. a) For every $x \in G$, Lemma $2.1 \mathrm{f}$ ) ensures $f(x)=f[f(x)]$, which implies $f(x-f(x))=f(0)=0$ by Lemma 2.3.

b) Let $x \in G$ be arbitrary. Then by a) $x=(x-f(x))+f(x) \in K_{f}+f(G)$, so $G=K_{f}+f(G)$. Let be $y \in K_{f} \cap f(G)$. $y \in K_{f}$ means $f(y)=0 ; y \in f(G)$ means by Lemma 2.1 e) $f(y)=y$, so in the total $y=0$, i.e. $K_{f} \cap f(G)=\{0\}$. For the uniqueness of $x=x_{1}+x_{2}, x_{1} \in K_{f}, x_{2} \in f(G)$, the usual argument in the case of two subgroups $K_{f}$ and $f(G)$ cannot be applied here because $f(G)$ need not be a subgroup of $G$. Assume that $x \in G, x=x_{1}+x_{2}=y_{1}+y_{2}$ with $x_{1}, y_{1} \in K_{f}, x_{2}, y_{2} \in f(G)$. Then $f\left(x_{2}\right)={ }_{(3)}=f\left(x_{1}+x_{2}\right)=f\left(y_{1}+y_{2}\right)={ }_{(3)}=f\left(y_{2}\right)$, so by Lemma $2.1 \mathrm{e}) x_{2}=y_{2}$ and finally also $x_{1}=y_{1}$.

c) If $x \in G, x=x_{1}+x_{2}, x_{1} \in K_{f}, x_{2} \in f(G)$, then $f(x)=f\left(x_{1}+x_{2}\right)={ }_{(3)}=$ $f\left(x_{2}\right)=($ L. $\left.2.1 \mathrm{e})\right)=x_{2} \in K_{f}+x_{2}=K_{f}+x_{1}+x_{2}=K_{f}+x$.

Example 2.7. Let be $G:=\mathbb{Z}_{2}^{3} \cong \mathbb{Z}_{2} \oplus \mathbb{Z}_{2} \oplus \mathbb{Z}_{2}$ the elementary abelian 2-group of dimension $3, e_{1}:=(1,0,0), e_{2}:=(0,1,0), e_{3}:=(0,0,1)$.

Let $f_{0}: G \longrightarrow G$ be defined by means of the following table:

\begin{tabular}{c|cccccccc}
$x$ & 0 & $e_{1}$ & $e_{2}$ & $e_{3}$ & $e_{1}+e_{2}$ & $e_{2}+e_{3}$ & $e_{1}+e_{3}$ & $e_{1}+e_{2}+e_{3}$ \\
\hline$f_{0}(x)$ & 0 & 0 & $e_{2}$ & $e_{3}$ & $e_{2}$ & $e_{1}+e_{2}+e_{3}$ & $e_{3}$ & $e_{1}+e_{2}+e_{3}$
\end{tabular}

It can be immediately checked that

$$
\begin{array}{ll}
y+f_{0}(y) \in\left\{0, e_{1}\right\}=K_{f_{0}}(\forall y \in G), \\
f_{0}\left(x+e_{1}\right)=f_{0}(x) & (\forall x \in G) .
\end{array}
$$

For arbitrary $x, y \in G$ we get $x+y+f_{0}(y) \in_{(4)} \in\left\{x, x+e_{1}\right\}$, so $f_{0}(x+y+$ $\left.f_{0}(y)\right) \in\left\{f_{0}(x), f_{0}\left(x+e_{1}\right)\right\}={ }_{(5)}=\left\{f_{0}(x)\right\}$, i.e., $f_{0}\left(x+y+f_{0}(y)\right)=f_{0}(x)=$ $f_{0}(x)+2 f_{0}(y)$, so $f_{0} \in S_{0}(G)$, but $f_{0}$ is not additive: $f_{0}\left(e_{2}+e_{3}\right)=e_{1}+e_{2}+e_{3} \neq$ $e_{2}+e_{3}=f_{0}\left(e_{2}\right)+f_{0}\left(e_{3}\right)$. Moreover, $K_{f_{0}}=\left\{0, e_{1}\right\}$ is a subgroup of $G$, but $f_{0}(G)=\left\{0, e_{2}, e_{3}, e_{1}+e_{2}+e_{3}\right\}$ is not. However, $G=K_{f_{0}} \oplus f_{0}(G)$, so, together 
with (5), Lemmas 2.4 a) and $2.6 \mathrm{~b})$ are confirmed. (4) and $y-f_{0}(y)=y+f_{0}(y)$ $(\forall y \in G)$ ensure Lemma 2.6 a).

This example shows moreover that if $G$ is an abelian group, $A$ a subgroup of $G, B$ a subset of $G$ with $G=A \oplus B$, then $B$ need not be a subgroup of $G$. by

The great importance of $K_{f}:=f^{-1}(\{0\})$ und $f(G)$ for $f \in S_{0}(G)$ is underlined

Corollary 2.8. $f_{1}, f_{2} \in S_{0}(G) \Longrightarrow\left[f_{1}=f_{2} \Longleftrightarrow K_{f_{1}}=K_{f_{2}} \wedge f_{1}(G)=f_{2}(G)\right]$.

Proof. $\Longrightarrow$ is trivial. $\Longleftarrow: K:=K_{f_{1}}=K_{f_{2}}$. Let be $x \in G$ arbitrary. By Lemma $2.6 \mathrm{~b})$ there exist $x_{1} \in K, x_{2} \in f_{1}(G)=f_{2}(G)$ with $x=x_{1}+x_{2}$, so $\left.f_{1}(x)=f_{1}\left(x_{1}+x_{2}\right)={ }_{(3)}=f_{1}\left(x_{2}\right)={ }_{(\text {L. } 2.1 ~ \mathrm{e})}\right)=x_{2}=f_{2}\left(x_{2}\right)=f_{2}\left(x_{1}+x_{2}\right)=f_{2}(x)$, i.e., $f_{1}=f_{2}$.

Next, a connection to the functional equation

$$
\begin{gathered}
f: F \longrightarrow F ; a, b, c, p, q, r \in F, a b p q \neq 0, \\
f(a x+b y+c)=p f(x)+q f(y)+r(\forall x, y \in F)
\end{gathered}
$$

becomes visible. The beautiful theory of (DL) has been developed by Z. Daróczy [4] and L. Losonczi [9] in their Ph.D. works supervised by J. Aczél for a field $F$ of characteristic 0. A very modest side case of (DL) occurs in Lemma 2.9 below ( $a=p=1, b=q=2, c=r=0)$, but $(G,+)$ need not be the additive group of a field nor will it have a priori any divisibility properties such as $(F,+)$ does in the most perfect sense: it is divisible and torsion-free.

Lemma 2.9. If $f \in S_{0}(G)$, then

$$
f(x+2 y)=f(x)+2 f(y) \quad(\forall x, y \in G) .
$$

The converse is false.

Proof. 1) Let be $x, y \in G$ arbitrary. By Lemma 2.6 a) $y-f(y) \in K_{f}$, so $f(x+2 y)=$ $\left.f(x+y+f(y)+y-f(y))={ }_{(3)}=f(x+y+f(y))={ }_{(1)}=f(x)+2 f(y) .-2\right)$ For $G=\mathbb{Q}, f:=2 i_{\mathbb{Q}}$ is a solution of $(6)$, but not of $\left(1_{0}\right):$ it is not idempotent. This expresses the contrast between linearity of $(6)$ and nonlinearity of $\left(1_{0}\right)$.

Remark 2.10. For abelian groups $A, B$ and the linear functional equation

$$
h: A \longrightarrow B, h(x+2 y)=h(x)+2 h(y) \quad(\forall x, y \in A) ; h(0)=0
$$

we state without proof some important properties of solutions $h$ of $\left(6_{0}^{\prime}\right)$ the analogues of which for $f \in S_{0}(G)$ are indicated in brackets.

a) $h$ is $\mathbb{Z}$-homogeneous and a fortiori odd, but not additive in general (Lemma 2.13 b), Example 2.7). 
b) $2 h$ is additive (Lemma $2.13 \mathrm{i})$ ).

c) If $\omega_{2}: A \longrightarrow A$ is surjective, then $h$ is additive (Theorem 3.2; the proof of [1], p. 66 , Theorem 1 , is easily adapted to the present situation).

d) If $\omega_{2}: B \longrightarrow B$ is injective, then $h$ is additive (Theorem 3.9).

Lemma 2.11. For $f \in S(G)$ we have:

a) $i_{G}-f$ idempotent $\Longleftrightarrow f(0)=0$.

b) $\left(i_{G}-f\right) \in S(G) \Longleftrightarrow f$ is additive.

Proof. a) For every $x \in G$ we obtain $\left(i_{G}-f\right) \circ\left(i_{G}-f\right)(x)=\left(i_{G}-f\right)(x-f(x))=$ $x-f(x)-f(x-f(x))=\left(\right.$ L. 2.1 f),L. 2.3) $=\left(i_{G}-f\right)(x)-f(0)$, and the assertion follows at once from this identity.

b) Case 1: $f(0) \neq 0$. Then $f$ is not additive, and by a) $\left(i_{G}-f\right)$ is not idempotent, so by Lemma $2.1 \mathrm{f})\left(i_{G}-f\right) \notin S(G)$, and assertion b) holds here.

Case 2: $f(0)=0$. We put $g:=i_{G}-f$ and get for all $x, y \in G: g(x+y+g(y))=$ $x+y+g(y)-f(x+y+g(y))=x+y+y-f(y)-f(x+y+y-f(y))=$ $x+2 y-f(y)-f((x+y)+(y-f(y)))={ }_{(\text {Case } 2 \text {; L. 2.6 a }))}=x+2 y-f(y)-f((x+y)$ $+(y-f(y))+f(y-f(y)))={ }_{(1)}=x+2 y-f(y)-f(x+y)-2 f(y-f(y))=$ $x+2 y-f(y)-f(x+y)=(x-f(x))+2(y-f(y))-f(x+y)+f(x)+f(y)$, i.e.

$$
g(x+y+g(y))=g(x)+2 g(y)+f(x)+f(y)-f(x+y)(\forall x, y \in G) .
$$

So by $(7)\left(i_{G}-f\right)=g \in S(G)$ if and only if $f$ is additive.

Lemma 2.12. For $f \in S_{0}(G)$, the following statements are equivalent:

(i) $u, v \in f(G) \Longrightarrow u+v \in f(G)$.

(ii) $f(G)$ is a subgroup of $G$.

(iii) $f$ is additive.

By Lemma 2.1 $\mathrm{f}), f$ then is the projection of $G$ onto $f(G)$ along $K_{f}$, and $K_{f}$ and $f(G)$ are direct summands of $G$.

Proof. (i) $\Longrightarrow$ (iii): Let $x, y \in G$ be arbitrary. By Lemma 2.6 b) there exist $x_{1}, y_{1} \in K_{f}$ and $x_{2}, y_{2} \in f(G)$ with $x=x_{1}+x_{2}, y=y_{1}+y_{2}$, so $f(x+y)=$ $f\left(x_{1}+y_{1}+x_{2}+y_{2}\right)={ }_{(3)}=f\left(x_{2}+y_{2}\right)$. By (i) $x_{2}+y_{2} \in f(G)$, so by Lemma $\left.2.1 \mathrm{e}\right)$ $f\left(x_{2}+y_{2}\right)=x_{2}+y_{2}=f\left(x_{2}\right)+f\left(y_{2}\right)={ }_{(3)}=f\left(x_{1}+x_{2}\right)+f\left(y_{1}+y_{2}\right)=f(x)+f(y)$, i.e., (iii) holds.

(iii) $\Longrightarrow$ (ii) $\Longrightarrow$ (i) is trivial.

Lemma 2.13. If $f \in S_{0}(G)$, then:

a) $v \in f(G), n \in \mathbb{Z} \Longrightarrow n v \in f(G) \quad(f(G)$ is $\mathbb{Z}$-homogeneous $)$.

b) $x \in G, n \in \mathbb{Z} \Longrightarrow f(n x)=n f(x)$ ( $f$ is $\mathbb{Z}$-homogeneous; cf. Remark 2.10 a)).

c) $u, v \in f(G) ;, r, s \in \mathbb{Z} \Longrightarrow r u+2 s v \in f(G)$.

d) $\left(i_{G}-f\right)(G)=K_{f}$.

e) $f(G)=\left(i_{G}-f\right)^{-1}(\{0\})$. 
f) $\left(i_{G}-f\right)(n x)=n\left(i_{G}-f\right)(x)(\forall x \in G, \forall n \in \mathbb{Z})$.

g) $\left(i_{G}-f\right)(G)=\left\{y \in G ;\left(i_{G}-f\right)(y)=y\right\}$.

h) $x \in G$, ord $x=n \in \mathbb{N} \Longrightarrow$ ord $f(x) \mid n$.

i) $k \in \mathbb{Z} \Longrightarrow 2 k f \in \operatorname{End}(G)$.

Proof. a) For $S:=\{n \in \mathbb{Z} ; v \in f(G) \Longrightarrow n v \in f(G)\}$ we show $S=\mathbb{Z} .0 \in S$ since $0 v=0=f(0) \in f(G)$ for all $v \in f(G)$, and $1 \in S$ is trivial. Assume $n \in S, u \in f(G)$ arbitrary. By definition of $S, n u \in f(G)$, say $f(x)=n u$ for a suitable $x \in G$. Then $f(x+u+f(u))={ }_{(1)}=f(x)+2 f(u)={ }_{\text {L. } 2.1 ~ \mathrm{e}))}=f(x)+2 u=$ $n u+2 u=(n+2) u$, i.e., $(n+2) u \in f(G)$. As $u \in f(G)$ was arbitrary, $n+2 \in S$. $\left.\left.f(x-u+f(-u))={ }_{(1)}=f(x)+2 f(-u)={ }_{(\mathrm{L} . ~} 2.1 \mathrm{~g}\right)\right)=f(x)-2 f(u)=n u-2 u=$ $(n-2) u$, i.e., $(n-2) u \in f(G)$. Since $u \in f(G)$ was arbitrary, $n-2 \in S$. Together with $0,1 \in S$ we obtain $2 \mathbb{Z} \subset S, 2 \mathbb{Z}+1 \subset S$, so $S=\mathbb{Z}$.

b) For $x \in G, n \in \mathbb{Z}, x=x_{1}+x_{2}$ with $x_{1} \in K_{f}, x_{2} \in f(G)$ (Lemma 2.6 b) we get $f(n x)=f\left(n\left(x_{1}+x_{2}\right)\right)=f\left(n x_{1}+n x_{2}\right)$. Now $n x_{1} \in K_{f}$ by Lemma $\left.2.4 \mathrm{a}\right)$, and

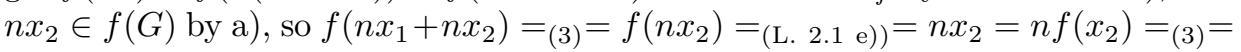
$n f\left(x_{1}+x_{2}\right)=n f(x)$.

c) There exist $x, y \in G$ with $f(x)=u, f(y)=v$. Then $f(r x+s y+f(s y))={ }_{(1)}=$ $f(r x)+2 f(s y)=_{\mathrm{b})}=r f(x)+2 s f(y)=r u+2 s v$, so $r u+2 s v \in f(G)$.

d) If $z \in\left(i_{G}-f\right)(G)$, then $z=\left(i_{G}-f\right)(x)=x-f(x)$ for some $x \in G$, and by Lemma 2.6 a) $z \in K_{f}$. Conversely, if $z \in K_{f}$, then $z-f(z)=z,\left(i_{G}-f\right)(z)=$ $z, z \in\left(i_{G}-f\right)(G)$.

e) For every $y \in G$ we have: $y \in f(G) \Longleftarrow_{(\text {L. } 2.1 \mathrm{e}))} \Longrightarrow f(y)=y \Longleftrightarrow\left(i_{G}-f\right)(y)$ $=0 \Longleftrightarrow y \in\left(i_{G}-f\right)^{-1}(\{0\})$.

f) $x \in G$ and $n \in \mathbb{Z}$ imply $\left(i_{G}-f\right)(n x)=n x-f(n x)=_{\mathrm{b})}=n x-n f(x)=$ $n\left(i_{G}-f\right)(x)$.

g) follows from Lemma 2.11 a) and Remark 1.2.

h) $n x=0$ implies $0=f(n x)={ }_{\mathrm{b})}=n f(x)$, so ord $f(x) \in \mathbb{N}$, ord $f(x) \mid n$.

i) Let be $x, y \in G$ arbitrary. Then $2 k f(x+y)=_{\mathrm{b})}=k f(2 x+2 y)={ }_{(\text {L. } 2.9)}=$ $k(f(2 x)+2 f(y))=_{\mathrm{b})}=k(2 f(x)+2 f(y))=2 k f(x)+2 k f(y)$.

Remark 2.14. The property of $f(G)$ in Lemma 2.13 c) does not imply that $f(G)$ is a subgroup of $G$; it cannot because of Example 2.7 where $f(G)=\left\{0, e_{2}, e_{3}, e_{1}+\right.$ $\left.e_{2}+e_{3}\right\}$.

The notion of purity in abelian group theory was introduced by H. Prüfer [11] for subgroups ("Servanzuntergruppe") and turned out to be extremely important and handy. We find it useful for our purposes to extend it to subsets rather than using it only for subgroups of our abelian group $G$. Purity says that the subset inherits a divisibility property from $G$ :

Definition 2.15. A subset $M$ of $G$ is called pure in $G$ or a pure subset of $G$ if for all $y \in M, n \in \mathbb{N}, n \mid y$ in $G$ implies $n \mid y$ in $M$, i.e., if the existence of $x \in G$ with $n x=y$ implies that of $x^{\prime} \in M$ with $n x^{\prime}=y$, i.e., if $M \cap n G \subset n M$. 
Lemma 2.16. For $f \in S_{0}(G)$ we have:

a) $f(G)$ is a pure subset of $G$.

b) $K_{f}$ is a pure subgroup of $G$.

Proof. a) Let be $y \in f(G), n \in \mathbb{N}, n \mid y$ in $G$. There exists $x \in G$ with $y=n x$,

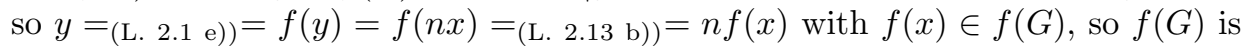
pure in $G$.

b) By Lemma 2.4 a), $K_{f}$ is a subgroup of $G$. Let be $y \in K_{f}, n \in \mathbb{N}, n \mid y$ in $G$. There exists $x \in G$ with $y=n x$, and by Lemma $2.13 \mathrm{~d}), y \in\left(i_{G}-f\right)(G)$. So $\left.y=(\mathrm{L} .2 .13 \mathrm{~g}))=\left(i_{G}-f\right)(y)=\left(i_{G}-f\right)(n x)=(\mathrm{L} .2 .13 \mathrm{f})\right)=n\left(i_{G}-f\right)(x)$ where $\left(i_{G}-f\right)(x) \in\left(i_{G}-f\right)(G)=($ L. $\left.2.13 \mathrm{~d})\right)=K_{f}$. Therefore $n \mid y$ in $K_{f}$, and $K_{f}$ is pure in $G$.

\section{Remark 2.17.}

a) For every abelian group $G$, the subgroups $\{0\}$ and $G$ are pure in $G$, which is immediate from Definition 2.15.

b) Definition: If $\{0\}$ and $G$ are the only pure subgroups of $G$, then $G$ is called pure-simple.

c) Purity is a relative property (exactly like openness and closedness for subsets of a topological space): Every subgroup $H$ of $G$ is pure in itself (cf. part a)) but not necessarily pure in $G$. This fact is emphasized by the formulation "pure in...".

d) An abelian group $G$ is pure-simple if and only if it is isomorphic to a subgroup of $(\mathbb{Q},+)$ or $\mathbb{Z}\left(p^{\infty}\right)$ for some $p \in \mathbb{P}([6]$, p. 119 , Ex.7). So $\mathbb{Z}$ is not pure in $\mathbb{Q}$, which is obvious.

e) By Lemma 2.16, $K_{f}$ and $f(G)$ are pure in $G$ for every $f \in S_{0}(G)$. This is not true for an arbitrary group endomorphism: Take $G:=\mathbb{Z}_{4}=\{0,1,2,3\}$, $f:=\omega_{2}: \mathbb{Z}_{4} \longrightarrow \mathbb{Z}_{4}$. Then $f \in \operatorname{End}(G), K_{f}=\{0,2\}=f(G)$. From $\mathbb{Z}_{4} \hookrightarrow \mathbb{Z}\left(2^{\infty}\right)$ it follows by d) that $\mathbb{Z}_{4}$ is pure-simple, so $\{0,2\}$ is not pure in $\mathbb{Z}_{4}$. This example also shows that purity in $G$ is not preserved under endomorphisms of $G$.

f) In the problem of computing $S_{0}(G)$, Lemma 2.16 often constitutes a helpful and efficient instrument, e.g. in the following Theorem 2.18.

g) It is clear that always $\left\{\underline{0}, i_{G}\right\} \subset S_{0}(G)$.

Theorem 2.18. If $G$ is pure-simple, then $S_{0}(G)=\left\{\underline{0}, i_{G}\right\}$. The converse is not true (cf. Remark 3.15 a)).

Proof. By Lemma 2.16, $K_{f}$ and $f(G)$ are pure in $G$ for all $f \in S_{0}(G)$. Therefore the only possible cases are, by Lemma $2.6 \mathrm{~b}$ ) and Def. $2.17 \mathrm{~b}), K_{f}=\{0\}, f(G)=G$ or $K_{f}=G, f(G)=\{0\}$. In the first case $f=i_{G}$ (by Lemma $2.1 \mathrm{e}$ )), and in the second case $f=\underline{0}$. Together with Remark $2.17 \mathrm{~g}$ ) the assertion follows. 
Theorem 2.18 already deals with a special class of abelian groups (pure-simple). This kind of results is the subject of the next section. We finish this section with a simple and useful tool for the case that, for $f \in S_{0}(G)$, the subgroup $K_{f}$ (Lemma 2.4 a)) has finite index in $G$.

Lemma 2.19. If $f \in S_{0}(G)$ and $K_{f}$ has finite index $k$ in $G$, then:

a) $v \in f(G) \Longrightarrow$ ord $v \leq k$.

b) If there exists $v \in f(G)$ with ord $v=k$, then $f$ is additive.

c) $k=2$ implies additivity of $f$.

Proof. By definition, $k=$ card $\left(G / K_{f}\right)$, by Lemma 2.4 a) card $f(G) \leq k$, and by Lemma 2.4 c) $k \leq$ card $f(G)$, hence card $f(G)=k$.

a) By Lemma 2.13 a) $\langle v\rangle \subset f(G)$, so ord $v=\operatorname{card}\langle v\rangle \leq \operatorname{card} f(G)=k$.

b) ord $v=k$ means $\langle v\rangle=f(G)$, and by Lemma 2.12, $f$ is additive.

c) By a) ord $v \leq k=2$ for every $v \in f(G)$. Since card $f(G)=k=2$, there must exist $v \in f(G)$ with ord $v=2$, and additivity of $f$ follows from b).

\section{Remark 2.20.}

a) In Example $2.7, k=$ card $f_{0}\left(\mathbb{Z}_{2}^{3}\right)=4$ but ord $v \leq 2\left(\forall v \in \mathbb{Z}_{2}^{3}\right)$. Hence Lemma $2.19 \mathrm{a}$ ) is verified while the hypothesis of Lemma $2.19 \mathrm{~b})$ is not satisfied, and accordingly $f_{0}$ is not additive.

b) Lemma 2.19 c) can be read as follows: If $f \in S_{0}(G)$ exists and if $\operatorname{card} f(G)=$ $k=2$, then $f$ must be additive. But $k=2$ alone implies by no means the existence of an $f \in S_{0}(G)$ with card $f(G)=2: G:=\mathbb{Z}_{4}$ is pure-simple (cf. Remark 2.17 e)), so by Theorem $2.18, S_{0}(G)$ cannot contain an element $f$ with $\operatorname{card} f(G)=2$.

\section{Results for special classes of abelian groups}

\section{Definition 3.1.}

a) If $p \in \mathbb{P}, G$ is called $p$-divisible iff $G=p G$. (Of course every divisible $G$ is $p$-divisible.)

b) $G$ is called uniquely $p$-divisible iff $\omega_{p}: G \longrightarrow G$ is bijective.

c) A subset $M$ of $G$ is called p-divisible iff $\omega_{p}(M) \subset M$ and the restriction $\tilde{\omega}_{p}: M \longrightarrow M$ of $\omega_{p}$ is surjective.

Theorem 3.2. If $G$ is 2-divisible, then $S_{0}(G) \subset \operatorname{End}(G)(c f$. Remark 2.10 c)).

Proof. Let be $f \in S_{0}(G)$ and $x, y \in G$ arbitrary. Since $G=2 G$, there exist $v, w \in G$ with $2 v=x, 2 w=y$. It follows that $f(x+y)=f(2 v+2 w)=$ $f(2(v+w))=($ L. $2.13 \mathrm{~b}))=2 f(v+w)={ }_{(\mathrm{L} .2 .13 \mathrm{i}))}=2 f(v)+2 f(w)=f(2 v)+f(2 w)=$ $f(x)+f(y)$. Since $x, y$ were arbitrary, we have $f \in \operatorname{End}(G)$. 
In favor of a possibly higher flexibility in the applications, we state an "individualized" version of Theorem 3.2 :

Theorem 3.3. If $\in S_{0}(G)$ and $f(G)$ is 2-divisible, then $f$ is additive.

Proof. We first note that by Lemma 2.13 a), $\omega_{2}[f(G)] \subset f(G)$ automatically holds. Let $u, v \in f(G)$ be arbitrary. By Definition 3.1c) there exist $u^{\prime}, v^{\prime} \in f(G)$ with $2 u^{\prime}=u, 2 v^{\prime}=v$, so there exist $x, y \in G$ with $f(x)=u^{\prime}, f(y)=v^{\prime}$. Therefore $u+v=2 u^{\prime}+2 v^{\prime}=2 f(x)+2 f(y)=($ L. 2.13 i $\left.)\right)=2 f(x+y)=($ L. 2.13 b) $)=f(2(x+y)) \in$ $f(G)$, so $u+v \in f(G)$. As $u, v \in f(G)$ were arbitrary, additivity of $f$ follows from Lemma 2.12 .

Next we look at locally cyclic groups $G$.

Definition 3.4. $G$ is called locally cyclic iff every finitely generated subgroup of $G$ is cyclic. (Clearly such a group is automatically abelian, and every cyclic group is locally cyclic.)

Remark 3.5. $G$ is locally cyclic if and only if one of the following pairwise equivalent conditions holds:

(i) $G$ is a direct limit of cyclic groups ([6], p. 58, Exercise 1 b)).

(ii) $G$ is isomorphic to a subgroup of $(\mathbb{Q},+)$ or $(\mathbb{Q} / \mathbb{Z},+)([14]$, p. 194 , Theorem 1).

(iii) The lattice of subgroups of $G$ is distributive (Theorem of Ore, [10], p. 267, Theorem 4).

By the way, (ii), $\mathbb{Q} / \mathbb{Z} \cong \oplus_{p \in \mathbb{P}} \mathbb{Z}\left(p^{\infty}\right)$, and Remark $2.17 \mathrm{~d}$ ) imply that every pure-simple $G$ is locally cyclic, so that we may expect some extension of Theorem 2.18 .

Theorem 3.6. If $G$ is locally cyclic, then $S_{0}(G) \subset \operatorname{End}(G)$, but in general $S_{0}(G) \neq$ $\left\{\underline{0}, i_{G}\right\}$.

Proof. 1) Let be $f \in S_{0}(G)$ and $x, y \in G$ arbitrary. By assumption, there exists $d \in G$ with $\langle\{x, y\}\rangle=\langle d\rangle$, so $x=r d, y=s d$ for some $r, s \in \mathbb{Z}$, and $f(x+y)=$ $f(r d+s d)=f((r+s) d)=($ L. 2.13 b $))=(r+s) f(d)=r f(d)+s f(d)=f(r d)+f(s d)=$ $f(x)+f(y)$. Since $x, y$ were arbitrary, $f \in \operatorname{End}(G)$, i.e., $S_{0}(G) \subset \operatorname{End}(G)$.

2) $G:=\mathbb{Z}_{6}=\{0,1,2,3,4,5\}$ is locally cyclic, and $G \cong \mathbb{Z}_{2} \oplus \mathbb{Z}_{3}$. The subgroups of $\mathbb{Z}_{6}$, namely $\{0\},\{0,3\},\{0,2,4\}, Z_{6}$, form a distributive lattice (cf. Remark 3.5 (iii)). All of them are pure in $\mathbb{Z}_{6}$, as direct summands of $\mathbb{Z}_{6}([7]$, p. $14(\mathrm{~h}))$. By part 1) of this proof, $S_{0}\left(\mathbb{Z}_{6}\right) \subset \operatorname{End}\left(\mathbb{Z}_{6}\right)$. It is well known that $\operatorname{End}\left(\mathbb{Z}_{6}\right)=\left\{\omega_{k} ; k \in\right.$ $\{0,1,2,3,4,5\}\}$, and precisely $\omega_{0}=\underline{0}, \omega_{1}=i_{Z_{6}}, \omega_{3}$ and $\omega_{4}$ are idempotent, hence by Lemma $2.1 \mathrm{f}$ ) and by $(2) \Longrightarrow(1)$ we have $S_{0}\left(\mathbb{Z}_{6}\right)=\left\{\omega_{0}, \omega_{1}, \omega_{3}, \omega_{4}\right\}$, 
i.e., $S_{0}\left(Z_{6}\right)$ consists of the projections of $\mathbb{Z}_{6}$ onto its four subgroups along the respective complementary subgroup, and $S_{0}(G)=\left\{\underline{0}, i_{G}\right\}$ is violated.

The next aspect we are going to consider is the non-existence of elements of order 2 in $G$, i.e., the case where $\omega_{2}: G \longrightarrow G$ is injective (cf. Remark 1.3).

Definition 3.7. $G$ is called 2-torsion-free (sometimes 2-cancellable) if $\omega_{2}: G \longrightarrow G$ is injective. (Notice that then $S(G)=S_{0}(G)$ by Lemma 2.2.) Evidently every torsion-free $G$ and a fortiori every free abelian $G$ is 2-torsion-free.

Lemma 3.8. If $G$ is arbitrary, if $f \in S_{0}(G)$ and if

$$
w \in G, 2 w \in f(G) \Longrightarrow w \in f(G)
$$

then $f$ is additive.

Proof. Let be $u, v \in f(G)$ arbitrary. Then by Lemma $2.13 \mathrm{c}$ ) with $r=2, s=1$, we get $2(u+v)=2 u+2 v \in f(G)$, so by $(8) u+v \in f(G)$, and by Lemma $2.12 f$ is additive.

Theorem 3.9. $G$ 2-torsion-free implies $S_{0}(G) \subset \operatorname{End}(G)(c f$. Remark $\left.2.10 \mathrm{~d})\right)$.

Proof. Assume $f \in S_{0}(G)$ and $w \in G, 2 w \in f(G)$. By Lemma 2.1 e) $2 w=f(2 w)$, so by Lemma 2.13 b) $2 w=2 f(w)$. Injectivity of $\omega_{2}$ yields $w=f(w) \in f(G)$. This means that (8) holds, and by Lemma $3.8 f$ is additive, i.e. $S_{0}(G) \subset \operatorname{End}(G)$.

As with Theorems 3.2 and 3.3, we present an individualized and sharpened version of Theorem 3.9:

Theorem 3.10. If $f \in S_{0}(G)$ and if $K_{f}$ contains no element of order 2 , then $f$ is additive.

Proof. Let be $w \in G, 2 w \in f(G)$. Then $2 w=_{(\text {L.2.1 e }))}=f(2 w)={ }_{(\text {L. } 2.13 \text { b }))}=2 f(w)$, hence $2(w-f(w))=0$. By Lemma 2.6 a) $w-f(w) \in K_{f}$, and the assumption on $K_{f}$ leads to $w-f(w)=0, w=f(w) \in f(G)$, i.e., (8) holds, and the assertion follows from Lemma 3.8.

Remark 3.11. (8) need not hold when $f(G)$, instead of $K_{f}$, has no element of order 2: $G:=\mathbb{Z}_{2}=\{0,1\}, f=\underline{0}, w:=1$. This shows that (8) is sufficient but not necessary for $f$ to be additive.

We terminate this section by considering indecomposability of $G$.

Definition 3.12. $G$ is said to be (directly) indecomposable if for any subgroups $A, B$ of $G, G=A \oplus B$ implies $A=\{0\}$ or $B=\{0\}$. 
Remark 3.13. If $G$ is indecomposable, then $G$ cannot be a mixed group, i.e., it must be a torsion group or torsion-free ([7], p. 22, Theorem 10). We establish now a connection to our functional equation $\left(1_{0}\right)$ :

Theorem 3.14. For the conditions

(i) $G$ is pure-simple,

(ii) $G$ is indecomposable,

(iii) $S_{0}(G)=\left\{\underline{0}, i_{G}\right\}$,

we have (i) $\Longleftrightarrow$ (ii) $\Longleftrightarrow$ (iii).

Proof. (i) $\Longrightarrow$ (ii): If there were subgroups $A, B \neq\{0\}$ with $G=A \oplus B$, then $A, B$ would be pure in $G$ ([7], p. $14(\mathrm{~h})$ ), a contradiction to (i).

(ii) $\Longrightarrow$ (i): Let for some $p \in \mathbb{P}$ the group $G$ be the additive group $\left(J_{p},+\right)$ of $p$-adic integers. By a theorem of $R$. Baer, $J_{p}$ is indecomposable ([7], p. 46, Theorem 18). If $J_{p}$ were pure-simple, it would be embeddable into a countable group by Remark $2.17 \mathrm{~d}$ ), contradicting the fact that $J_{p}$ is uncountable. Therefore (i) is violated.

(ii) $\Longrightarrow$ (iii): Case 1: $G$ is not torsion-free. By Remark $3.13, G$ is a torsion group, and by [7], p. 22 , Theorem 10 , it is embeddable into $\mathbb{Z}\left(p^{\infty}\right)$ for some $p \in \mathbb{P}$, so pure-simple by Remark $2.17 \mathrm{~d}$ ). Now (iii) follows from Theorem 2.18.

Case 2: $G$ is torsion-free. By Theorem 3.9, $S_{0}(G) \subset \operatorname{End}(G)$. Let be $f \in S_{0}(G)$ arbitrary. Then $f(G)$ is a subgroup of $G$, and by Lemma 2.6 b) $G=K_{f} \oplus f(G)$, which is here a decomposition into subgroups. By (ii) $f(G)=\{0\}$ or $f(G)=G$, i.e., $f=\underline{0}$ or, by Lemma $2.1 \mathrm{e}$ ), $f=i_{G}$, and (iii) holds again.

(iii) $\Longrightarrow$ (ii): If there were subgroups $A, B \neq\{0\}$ of $G$ with $G=A \oplus B$, then the projection $f_{1}$ of $G$ onto $A$ along $B$ would be additive and idempotent, hence in $S_{0}(G)$, a contradiction to (iii).

\section{Remark 3.15.}

a) Theorem 3.14 (iii) $\Longrightarrow$ (i) shows that the converse of Theorem 2.18 is not true.

b) The group $J_{p}$ of $p$-adic integers is not pure-simple. Therefore it must possess a pure subgroup $H$ with $\{0\} \neq H \neq J_{p}$. Since $S_{0}\left(J_{p}\right)=\left\{\underline{0}, i_{J_{p}}\right\}$ by Theorem 3.14, $H$ cannot be $K_{f}$ or $f(G)$ for an $f$ in $S_{0}\left(J_{p}\right)$. This shows that Lemma $2.16 \mathrm{a}), \mathrm{b})$ have no converses.

\section{Results for torsion groups}

So far the main question as to whether $S_{0}(G) \subset \operatorname{End}(G)$ has been answered for several classes of abelian groups. The answer is affirmative for $G$ torsion-free (Theorem 3.9) and a fortiori for the case $G=\mathbb{R}$ mentioned in the Introduction. 
We shall now focus on the case of a torsion group $G$ and begin by some auxiliary results.

Definition 4.1. If $G$ is an abelian group, $H$ a subgroup of $G, x \in H, p \in \mathbb{P}$, then $h_{p, H}(x):=\sup \left\{k \in \mathbb{N}^{0} ; p^{k} \mid x\right.$ in $\left.H\right\}$ ( $\infty$ allowed) is called the $p$-height of $x$ relative to the subgroup $H$.

It is clear from Definitions 2.15 and 4.1 that for a pure subgroup $H$ of $G$ and $x \in H$ we have $h_{p, H}(x)=h_{p, G}(x)$, in which case we merely write $h_{p}(x)$.

Lemma 4.2. ([7], p. 21, Lemma 8.) If $p \in \mathbb{P}$ and if $G$ is a p-group such that $x \in G$ and ord $x=p$ imply $h_{p}(x)=\infty$, then $G$ is divisible. (For arbitrary abelian groups $G$ this is not true: consider $\mathbb{Z}$ or $\mathbb{Z}\left(p^{\infty}\right) \oplus \mathbb{Z}$.)

The next lemma is one of the splitting theorems of Kulikov.

Lemma 4.3. ([8], p. 180, Lemma.) If $p \in \mathbb{P}$, if $G$ is a p-group and if $x \in G$, $\operatorname{ord} x=p, h_{p}(x)=k \in \mathbb{N}^{0}$, then there exists $y \in G$ with ord $y=p^{k+1}, x \in\langle y\rangle \cong$ $\mathbb{Z}_{p^{k+1}}$ and $G=\langle y\rangle \oplus C$ for an appropriate subgroup $C$ of $G$.

Theorem 4.4. For $k \in \mathbb{N}, H$ a 2-divisible subgroup of $G, G=\mathbb{Z}_{2^{k}} \oplus H$ we have $S_{0}(G) \subset \operatorname{End}(G)$.

Proof. We use $G \cong \mathbb{Z}_{2^{k}} \times H$ and write the elements of $G$ as ordered pairs (cf. Remark 1.1 and the Notation).

1) By hypothesis $2^{k} H=H$, so $2^{k}(\{0\} \times H)=\{0\} \times H$ for the given $k$. Let be $f \in S_{0}(G)$ arbitrary. By Lemma 2.13 b), $f$ is $\mathbb{Z}$-homogeneous, therefore

$$
f(\{0\} \times H)=f\left(2^{k}(\{0\} \times H)\right)=2^{k} f(\{0\} \times H) .
$$

Let be $\left(\eta_{1}, \eta_{2}\right) \in f(\{0\} \times H)$ arbitrary. Then by $(9)\left(\eta_{1}, \eta_{2}\right) \in 2^{k} f(\{0\} \times H)$, so there exists $\left(\zeta_{1}, \zeta_{2}\right) \in f(\{0\} \times H)$ with $\left(\eta_{1}, \eta_{2}\right)=2^{k}\left(\zeta_{1}, \zeta_{2}\right)=\left(2^{k} \zeta_{1}, 2^{k} \zeta_{2}\right)$ $=\left(0,2^{k} \zeta_{2}\right) \in\{0\} \times H$, so $f(\{0\} \times H) \subset\{0\} \times H$. This guarantees that the restriction $f_{2}:\{0\} \times H \longrightarrow\{0\} \times H$ of $f$ exists, and clearly $f_{2} \in S_{0}(\{0\} \times H)$, so by Theorem $3.2 f_{2} \in \operatorname{End}(\{0\} \times H)$, i.e.,

$f \in S_{0}(G) \Longrightarrow$ The restriction $f_{2}:\{0\} \times H \longrightarrow\{0\} \times H$ of $f$ is additive.

2) If $\xi_{0}$ is a generator of $\mathbb{Z}_{2^{k}}$, the general element of $G$ has the form $\left(l \xi_{0}, \xi\right)$ $(l \in \mathbb{Z}, \xi \in H) .2 H=H$ ensures the existence of $\xi^{\prime} \in H$ with $2 \xi^{\prime}=$ $\xi$. Let now again be $f \in S_{0}(G), l \in \mathbb{Z}, \xi \in H$ arbitrary. $f\left(l \xi_{0}, \xi\right)=$ $\left.f\left(\left(l \xi_{0}, 0\right)+(0, \xi)\right)=f\left(\left(l \xi_{0}, 0\right)+2\left(0, \xi^{\prime}\right)\right)={ }_{(6)}=f\left(l \xi_{0}, 0\right)+2 f\left(0, \xi^{\prime}\right)={ }_{(\mathrm{L} .2 .13 \mathrm{~b})}\right)$ $=l f\left(\xi_{0}, 0\right)+f(0, \xi)$, i.e.,

$$
f\left(l \xi_{0}, \xi\right)=l f\left(\xi_{0}, 0\right)+f_{2}(0, \xi) \quad\left(\forall f \in S_{0}(G), \forall l \in \mathbb{Z}, \forall \xi \in H\right) .
$$


3) Finally let be $f \in S_{0}(G) ; l, m \in \mathbb{Z} ; \xi, \eta \in H$ arbitrary. Then $f\left(\left(l \xi_{0}, \xi\right)+\right.$ $\left.\left.\left(m \xi_{0}, \eta\right)\right)=f\left((l+m) \xi_{0}, \xi+\eta\right)\right)={ }_{(11)}=(l+m) f\left(\xi_{0}, 0\right)+f_{2}(0, \xi+\eta)={ }_{(10)}=$ $(l+m) f\left(\xi_{0}, 0\right)+f_{2}(0, \xi)+f_{2}(0, \eta)={ }_{(11)}=f\left(l \xi_{0}, \xi\right)+f\left(m \xi_{0}, \eta\right)$, so $f \in$ $\operatorname{End}(G)$.

Remark 4.5. Caution: For $f \in S_{0}\left(\mathbb{Z}_{2^{k}} \oplus H\right)$, the inclusion $f\left(\mathbb{Z}_{2^{k}} \times\{0\}\right) \subset$ $\mathbb{Z}_{2^{k}} \times\{0\}$ need not hold: Put $H=\mathbb{Z}\left(2^{\infty}\right), \eta_{0} \in \mathbb{Z}\left(2^{\infty}\right)$, ord $\eta_{0}=2^{k}, f\left(l \xi_{0}, \xi\right):=$ $\left(0, l \eta_{0}+\xi\right)\left(\forall l \in \mathbb{Z}, \forall \xi \in \mathbb{Z}\left(2^{\infty}\right)\right.$. This contrast to the situation in the foregoing proof is mainly due to the fact that $H$ may contain an isomorphic copy of $\mathbb{Z}_{2^{k}}$ (as $\mathbb{Z}\left(2^{\infty}\right)$ does $)$. It can be shown however, that if $\omega_{2}: H \longrightarrow H$ is bijective, then $f\left(\mathbb{Z}_{2^{k}} \times\{0\}\right) \subset \mathbb{Z}_{2^{k}} \times\{0\}$ does hold.

Theorem 4.6. For $k, l \in \mathbb{N} G:=\mathbb{Z}_{2^{l}} \oplus \mathbb{Z}_{2^{k}}$ we have $S_{0}(G) \subset \operatorname{End}(G)$.

Proof. Again we use $G=\mathbb{Z}_{2^{l}} \times \mathbb{Z}_{2^{k}}$ in the sense of our notational convention. Without loss of generality we assume $l \leq k$.

1) We prove first

$$
\begin{gathered}
M \subset G \quad \mathbb{Z} \text {-homogeneous and pure in } G, \\
\varnothing \neq M \neq\{(0,0)\} \Longrightarrow \exists v \in M \text { with ord } v=2^{l} .
\end{gathered}
$$

In fact: Let be $\left(\xi^{\prime}, \eta^{\prime}\right) \in M \backslash\{(0,0)\}$, say ord $\left(\xi^{\prime}, \eta^{\prime}\right)=2^{m}$ where $1 \leq m \leq k$; notice that $\max \{\operatorname{ord} x ; x \in G\}=2^{k}$. $(\xi, \eta):=2^{m-1}\left(\xi^{\prime}, \eta^{\prime}\right) \in M$ since $M$ is $\mathbb{Z}$ homogeneous, and ord $(\xi, \eta)=2$. For a moment, we find the " $\mathbb{Q} / \mathbb{Z}$ notation" optimal: $\mathbb{Z}_{2^{l}}=\left\{0,1 / 2^{l}, \ldots,\left(2^{l}-1\right) / 2^{l}\right\}, \mathbb{Z}_{2^{k}}=\left\{0,1 / 2^{k}, \cdots,\left(2^{k}-1\right) / 2^{k}\right\}$. Now $(\xi, \eta)$ must be $(1 / 2,0)$ or $(0,1 / 2)$ or $(1 / 2,1 / 2)$, and we define $(\gamma, \delta):=\left(1 / 2^{l}, 0\right),\left(0,1 / 2^{l}\right)$ or $\left(1 / 2^{l}, 1 / 2^{l}\right)$ in the preceding three cases for $(\xi, \eta)$, respectively. Then in either case $(\xi, \eta)=2^{l-1}(\gamma, \delta)$, i.e., $2^{l-1} \mid(\xi, \eta)$ in $G$. Since $M$ is pure in $G$, we get $2^{l-1} \mid(\xi, \eta)$ in $M$, i.e., there exists $(\alpha, \beta) \in M$ with $2^{l-1}(\alpha, \beta)=(\xi, \eta) \neq(0,0)$. But $2^{l}(\alpha, \beta)=2(\xi, \eta)=(0,0)$, so ord $(\alpha, \beta)=2^{l}$, and (12) holds with $v:=(\alpha, \beta)$.

2) Let be $f \in S_{0}(G)$ arbitrary. If $f=(0,0)$ or $f=i_{G}$, then $f \in \operatorname{End}(G)$. So let be $f \notin\left\{(0,0), i_{G}\right\}$. Then $K_{f}$ and $f(G)$ are nonempty $\mathbb{Z}$-homogeneous pure subsets of $G$ (Lemmas 2.4 a), 2.13 a), 2.16) different from $\{(0,0)\}$. By (12)

both $K_{f}$ and $f(G)$ contain an element of order $2^{l}$.

$2^{l+k}=\operatorname{card} G=($ L. 2.6 b) $)=\operatorname{card}\left(K_{f} \oplus f(G)\right)=\operatorname{card} K_{f} \cdot \operatorname{card} f(G)$, therefore card $K_{f} \mid 2^{k+l}$, card $f(G) \mid 2^{k+l}$. If neither $K_{f}$ nor $f(G)$ contained an element of order $2^{k}$, then ord $u \mid 2^{k-1}\left(\forall u \in K_{f}\right)$ and ord $v \mid 2^{k-1}(\forall v \in f(G))$, so $\operatorname{ord}(u+v)|l \mathrm{~cm}(\operatorname{ord} u, \operatorname{ord} v)| 2^{k-1}$, i.e., ord $y \mid 2^{k-1}(\forall y \in G)$, in contradiction to $\max \{\operatorname{ord} x ; x \in G\}=2^{k}$. So

at least one of $K_{f}, f(G)$ contains an element of order $2^{k}$.

For facilitating the formulations, we put $\left\{M_{1}, M_{2}\right\}:=\left\{K_{f}, f(G)\right\}$. Without loss of generality, let be $v_{1} \in M_{1}$ with ord $v_{1}=2^{k}$ and $v_{2} \in M_{2}$ with ord $v_{2}=2^{l}$ 
(see(14) and (13)). $\mathbb{Z}$-homogeneity of $M_{1}, M_{2}$ implies $\left\langle v_{1}\right\rangle \subset M_{1},\left\langle v_{2}\right\rangle \subset M_{2}$, so

$$
\text { card } M_{1} \geq 2^{k} \text { and } \quad \text { card } M_{2} \geq 2^{l} \text {. }
$$

Now card $M_{1} \cdot$ card $M_{2}=$ card $G=2^{l+k}$, which makes strict inequality in (15) impossible, so $M_{1}=\left\langle v_{1}\right\rangle, M_{2}=\left\langle v_{2}\right\rangle$, and $f(G)$ must be a subgroup of $G$. By Lemma 2.12, $f \in \operatorname{End}(G)$.

Next we extend Example 2.7.

Lemma 4.7. If $A, B, C$ are abelian groups, $G:=A \oplus B \oplus C, D:=A \oplus 2 B \oplus 2 C$, $\alpha_{0} \in A$, ord $\alpha_{0}=2$, and if we define

$$
f: G \longrightarrow G, \quad f(\alpha, \beta, \gamma):=\left\{\begin{aligned}
(0, \beta, \gamma) & \text { if } x=(\alpha, \beta, \gamma) \in D \\
\left(\alpha_{0}, \beta, \gamma\right) & \text { if } x=(\alpha, \beta, \gamma) \in G \backslash D
\end{aligned}\right.
$$

then we have
a) $f \in S_{0}(G)$.
b) $K_{f}=A \oplus\{0\} \oplus\{0\}$.
c) If $2 B=B$ and $2 C=C$, then $f \in \operatorname{End}(G)$.
d) If $2 B \neq B$ and $2 C \neq C$, then $f \notin \operatorname{End}(G)$.

Proof. a) It follows at once from (16) that

$$
y+f(y) \in D \quad \forall y \in G) .
$$

Let be $x, y \in G$. If $x \in D, y \in G$, then by (17) $x+y+f(y) \in D$. If $x+y+f(y) \in D$, then $x=(x+y+f(y)-(y+f(y)) \in D$ by (17), so

$$
x, y \in G \Longrightarrow[x \in D \Longleftrightarrow x+y+f(y) \in D] .
$$

Inspecting the four cases for $x, y \in G$ to belong or not to belong to $D$ yields, with the help of (16) and (18), $f \in S_{0}(G)$.

b) follows immediately from (16).

c) We have $D=G$, and by (16) $f$ is the projection of $G$ onto $\{0\} \oplus B \oplus C$ along $K_{f}$, so $f \in \operatorname{End}(G)$.

d) Let be $\beta_{0} \in B \backslash 2 B, \gamma_{0} \in C \backslash 2 C$. Then $\left(\alpha_{0}, \beta_{0}, 0\right),\left(\alpha_{0}, 0, \gamma_{0}\right),\left(0, \beta_{0}, \gamma_{0}\right) \in$ $G \backslash D$, and $f\left(\alpha_{0}, \beta_{0}, 0\right)+f\left(\alpha_{0}, 0, \gamma_{0}\right)={ }_{(16)}=\left(\alpha_{0}, \beta_{0}, 0\right)+\left(\alpha_{0}, 0, \gamma_{0}\right)=\left(0, \beta_{0}, \gamma_{0}\right)$ while $f\left(\left(\alpha_{0}, \beta_{0}, 0\right)+\left(\alpha_{0}, 0, \gamma_{0}\right)\right)=f\left(0, \beta_{0}, \gamma_{0}\right)={ }_{(16)}=\left(\alpha_{0}, \beta_{0}, \gamma_{0}\right)$, so $f \notin \operatorname{End}(G)$.

The main results on $S_{0}(G)$ for abelian torsion groups $G$ is based upon the corresponding situation over their 2-components. So our next result concerns 2groups where of course divisibility and 2-divisibility are equivalent.

Theorem 4.8. For an abelian 2-group $G_{2}$, the following statements are equivalent:

(i) $S_{0}\left(G_{2}\right) \subset \operatorname{End}\left(G_{2}\right)$.

(ii) $G_{2}$ has the form $\left(\mathrm{ii}_{1}\right)$ or $\left(\mathrm{ii}_{2}\right)$ or $\left(\mathrm{ii}_{3}\right)$ where 
(ii 1 ) $G_{2}$ is 2-divisible,

(iii $)_{2} G_{2} \cong \mathbb{Z}_{2^{k}} \oplus C_{1}, k \in \mathbb{N}, C_{1}$ a 2-divisible abelian group,

(ii $\left.i_{3}\right) G_{2} \cong \mathbb{Z}_{2^{l}} \oplus \mathbb{Z}_{2^{k}}$ for some $k, l \in \mathbb{N}$.

Proof. (ii) $\Longrightarrow$ (i): (ii 1$) \Longrightarrow$ (i) by Theorem $3.2,\left(\mathrm{ii}_{2}\right) \Longrightarrow(\mathrm{i})$ by Theorem 4.4 , and $\left(\mathrm{ii}_{3}\right) \Longrightarrow$ (i) by Theorem 4.6 .

(i) $\Longrightarrow$ (ii). We prove the contrapositive $\neg$ (ii) $\Longrightarrow \neg(i)$, and $\neg$ (ii) means $\neg\left(\right.$ ii $\left._{1}\right) \wedge$ $\neg\left(\mathrm{ii}_{2}\right) \wedge \neg\left(\mathrm{ii}_{3}\right) . \neg\left(\mathrm{ii}_{1}\right)$ says that $G_{2}$ is not (2-)divisible. By Lemma 4.2 there exists $x \in G_{2}$ with ord $x=2$ and $h_{2}(x)=: k_{1} \in \mathbb{N}^{0}$. By Lemma 4.3

$$
G_{2} \cong \mathbb{Z}_{2^{k_{1}+1}} \oplus C_{1}
$$

for a suitable subgroup $C_{1}$ of $G_{2}$, uniquely determined up to isomorphism. Because of $\neg\left(\mathrm{ii}_{2}\right), C_{1}$ cannot be (2-)divisible. By Lemma 4.2 there exists $y \in C_{1}$ with ord $y=2$ and $h_{2, C_{1}}(y)=: k_{2} \in \mathbb{N}^{0}$. $C_{1}$ is a direct summand of $G_{2}$, so purity of $C_{1}$ in $G_{2}$ is guaranteed, and 2-height of $y$ in $C_{1}$ is the same as the one in $G_{2}$. By Lemma $4.3 C_{1} \cong \mathbb{Z}_{2^{k_{2}+1}} \oplus C_{2}$ for a suitable subgroup $C_{2}$ of $C_{1}$, therefore also of $G_{2}$. Together with (19) and the associativity of $\oplus$ we obtain

$$
G_{2} \cong \mathbb{Z}_{2^{k_{1}+1}} \oplus \mathbb{Z}_{2^{k_{2}+1}} \oplus C_{2}
$$

$\neg\left(\mathrm{ii}_{3}\right)$ now requires that $C_{2} \neq\{0\}$. As a subgroup of $G_{2}, C_{2}$ is a 2 -group and furthermore contains an element $z$ of order 2 . The first two summands in (20) are not 2-divisible, and from (20) and Lemma 4.7 a), d) $S_{0}\left(G_{2}\right) \backslash \operatorname{End}\left(G_{2}\right) \neq \varnothing$, i.e., $\neg(i)$ holds.

Our main result on $S_{0}(G)$ for torsion groups $G$ now reads as follows:

Theorem 4.9. For an abelian torsion group $G$ and its 2 -component $G_{2}$, the following statements are equivalent:

(a) $S_{0}(G) \subset \operatorname{End}(G)$,

(b) $S_{0}\left(G_{2}\right) \subset \operatorname{End}\left(G_{2}\right)$,

(c) $G_{2}$ has the form $\left(\mathrm{ii}_{1}\right)$ or $\left(\mathrm{ii}_{2}\right)$ or $\left(\mathrm{ii}_{3}\right)$ of Theorem 4.8 (ii).

Proof. (b) $\Longleftrightarrow$ (c) is Theorem 4.8.

(a) $\Longrightarrow$ (b): Let be $f_{2} \in S_{0}\left(G_{2}\right)$ arbitrary. For the $p$-components $G_{p}$ of $G$ $(p \in \mathbb{P}, p \neq 2)$ we put $f_{p}: G_{p} \longrightarrow G_{p}, f_{p}=\underline{0}$. Since $G=\oplus_{p \in \mathbb{P}} G_{p}$, every $x \in G$ has the form $x=\sum_{p \in \mathbb{P}} x_{p}$ with uniquely determined summands $x_{p} \in G_{p}(p \in \mathbb{P})$, all but finitely many being zero. We define

$$
f: G \longrightarrow G, f(x):=\sum_{p \in \mathbb{P}} f_{p}\left(x_{p}\right)=f_{2}\left(x_{2}\right)\left(\forall x=\sum_{p \in \mathbb{P}} x_{p} \in G\right) .
$$

Clearly $f(0)=0$, and $f$ is well-defined by (21). Let be $x, y \in G$ arbitrary. Then $f(x+y+f(y))=f\left(\sum x_{p}+\sum y_{p}+f\left(\sum y_{p}\right)\right)={ }_{(21)}=f\left(\sum x_{p}+\sum y_{p}+\sum f_{p}\left(y_{p}\right)\right)=$ $f\left(\sum\left(x_{p}+y_{p}+f_{p}\left(y_{p}\right)\right)\right)={ }_{(21)}=f_{2}\left(x_{2}+y_{2}+f_{2}\left(y_{2}\right)\right)={ }_{(1)}=f_{2}\left(x_{2}\right)+2 f_{2}\left(y_{2}\right)={ }_{(21)}=$ 
$f(x)+2 f(y)$, i.e., $f \in S_{0}(G)$, and by (a) $f \in \operatorname{End}(G)$. (21) also expresses that $f_{2}: G_{2} \longrightarrow G_{2}$ is the restriction of $f$, hence $f_{2} \in \operatorname{End}\left(G_{2}\right)$, and (b) holds.

(b) $\Longrightarrow\left(\right.$ a): Let be $f \in S_{0}(G)$ arbitrary. By Lemma $2.13 \mathrm{~h}$ ) we have $f\left(G_{2}\right) \subset$ $G_{2}$ as well as $f(H) \subset H$ for $H:=\oplus_{p \in \mathbb{P} \backslash\{2\}} G_{p}$. The restrictions $f_{2}: G_{2} \longrightarrow G_{2}$ and $f_{H}: H \longrightarrow H$ of $f$ are in $S_{0}(G), S_{0}(H)$, respectively. By (b) $f_{2} \in \operatorname{End}\left(G_{2}\right)$ and, since $H$ is 2-divisible, $f_{H} \in \operatorname{End}(H)$ by Theorem 3.2. Let be $x \in G=G_{2} \oplus H$ arbitrary, say $x=x_{2}+x_{H}=x_{2}+2 x_{H}^{\prime} \in G_{2} \oplus 2 H=G_{2} \oplus H=G$. Then $f(x)=f\left(x_{2}+2 x_{H}^{\prime}\right)=_{(\text {L. 2.9) }}=f\left(x_{2}\right)+2 f\left(x_{H}^{\prime}\right)={ }_{\text {L. } 2.13 \mathrm{~b})}=f\left(x_{2}\right)+f\left(2 x_{H}^{\prime}\right)=$ $f\left(x_{2}\right)+f\left(x_{H}\right)=f_{2}\left(x_{2}\right)+f_{H}\left(x_{H}\right)$, and $f_{2} \in \operatorname{End}\left(G_{2}\right), f_{H} \in \operatorname{End}(H)$ ensure $f \in \operatorname{End}(G)$, i.e., (a) holds.

\section{Concluding remarks}

Remark 5.1. The techniques used in parts (a) $\Longrightarrow(\mathrm{b})$ and (b) $\Longrightarrow$ (a) of the proof of Theorem 4.9 are merely special cases of those for a systematic treatment of the functional equation (1) over general direct sums of abelian groups.

Remark 5.2. A brief sketch for a prominent example of a direct sum is given: $G:=\mathbb{Q} / \mathbb{Z} \cong \oplus_{p \in \mathbb{P}} \mathbb{Z}\left(p^{\infty}\right) . \quad \mathbb{Q} / \mathbb{Z}$ is divisible and locally cyclic (cf. Remark 3.5), so that Theorems 3.2 or 3.6 ensure $S_{0}(\mathbb{Q} / \mathbb{Z}) \subset \operatorname{End}(\mathbb{Q} / \mathbb{Z})$. For every $p \in \mathbb{P}$ the $p$-component of $\mathbb{Q} / \mathbb{Z}$ is $\mathbb{Z}\left(p^{\infty}\right)$, and for every $f \in S_{0}(\mathbb{Q} / \mathbb{Z})$, Lemma $2.13 \mathrm{~h}$ ) implies

$$
f\left(\mathbb{Z}\left(p^{\infty}\right)\right) \subset \mathbb{Z}\left(p^{\infty}\right) \quad(\forall p \in \mathbb{P}),
$$

hence the restrictions $f_{p}: \mathbb{Z}\left(p^{\infty}\right) \longrightarrow \mathbb{Z}\left(p^{\infty}\right)(p \in \mathbb{P})$ of $f$ are available, and $f_{p} \in S_{0}\left(\mathbb{Z}\left(p^{\infty}\right)\right)(\forall p \in \mathbb{P})$. Additivity of $f$ leads to

$$
f(x)=\sum_{p \in \mathbb{P}} f_{p}\left(x_{p}\right) \quad\left(\forall x=\sum x_{p} \in \mathbb{Q} / \mathbb{Z}\right) .
$$

But now all the $\mathbb{Z}\left(p^{\infty}\right)(p \in \mathbb{P})$ are pure-simple (cf. Remark $2.17 \mathrm{~d}$ )), and from Theorem 2.18 we get $f_{p} \in\left\{\underline{0}, i_{\mathbb{Z}\left(p^{\infty}\right)}\right\}$, so that the general solution $f \in S_{0}(\mathbb{Q} / \mathbb{Z})$ appears in the lucid form $\mathbb{P}_{0}(f):=\left\{p \in \mathbb{P} ; f_{p}=\underline{0}\right\}, \mathbb{P}_{1}(f):=\left\{p \in \mathbb{P} ; f_{p}=i_{\mathbb{Z}\left(p^{\infty}\right)}\right\}$, $f(x)=\sum_{p \in \mathbb{P}_{1}(f)} x_{p}\left(\forall x=\sum x_{p} \in \mathbb{Q} / \mathbb{Z}\right)$. So $f$ is the projection of $\mathbb{Q} / \mathbb{Z}$ onto $f(\mathbb{Q} / \mathbb{Z})=\oplus_{p \in \mathbb{P}_{1}(f)} \mathbb{Z}\left(p^{\infty}\right)$ along $K_{f}=\oplus_{p \in \mathbb{P}_{0}(f)} \mathbb{Z}\left(p^{\infty}\right)$.

Remark 5.3. For torsion-free abelian groups $G$, Theorem 3.9 guarantees $S_{0}(G) \subset$ $\operatorname{End}(G)$, while for abelian torsion groups, Theorem 4.9 provides necessary and sufficient conditions for $S_{0}(G) \subset \operatorname{End}(G)$. For mixed abelian groups $G$ we have to expect much more complicated circumstances. Thus, e.g., in general no analogue of (22) and the subsequent decomposition (23) of $f \in S_{0}(G)$ will occur (cf. also Remark 4.5). We finish by an example which lies outside the scopes of Theorems $3.2,3.6,3.9$, and 3.14 . 
Example 5.4. $G:=\mathbb{Z}_{2} \oplus \mathbb{Z}$ is a splitting mixed abelian group; we write $\mathbb{Z}_{2}=\{0,1\}$. Then $(1,0)$ is the only element of $G$ of order 2 , and for any $f \in S_{0}(G)$, Lemma $2.13 \mathrm{~h}$ ) implies $f(1,0) \in\{(0,0),(1,0)\}$. If $f(1,0)=(0,0)$, then $f(1, \beta)=($ L. 2.4 a) $)=f(0, \beta)=_{\text {L. } 2.13 \text { b) })}=\beta f(1,0)(\forall \beta \in \mathbb{Z})$, i.e., $f$ is additive. If $f(1,0)=(1,0)$, then $(1,0) \notin K_{f}$, and $K_{f}$ does not contain an element of order 2 , so by Theorem $3.10 f$ is again additive. In the total $S_{0}\left(\mathbb{Z}_{2} \oplus \mathbb{Z}\right) \subset \operatorname{End}\left(\mathbb{Z}_{2} \oplus \mathbb{Z}\right)$. The six elements of $S_{0}\left(\mathbb{Z}_{2} \oplus \mathbb{Z}\right)$ are: $\underline{0}, i_{\mathbb{Z}_{2} \oplus \mathbb{Z}}$, the projections $(\varepsilon, \beta) \longmapsto(0, \beta)$ and $(\varepsilon, \beta) \longmapsto(\varepsilon, 0)$, and finally the mappings $(\varepsilon, \beta) \longmapsto(\beta \cdot 1, \beta),(\varepsilon, \beta) \longmapsto(\varepsilon+\beta \cdot 1,0)$, where $(\varepsilon, \beta) \in \mathbb{Z}_{2} \oplus \mathbb{Z}$. The last two solutions show that $f(\{0\} \times \mathbb{Z}) \subset\{0\} \times \mathbb{Z}$ is not valid in general.

\section{References}

[1] J. ACZÉL, Lectures on functional equations and their applications, Academic Press, New York-London, 1966.

[2] J. Aczél And J. Dhombres, Functional Equations in several variables, Cambridge University Press, Cambridge, 1989.

[3] W. W. Chaо, A projection in disguise, The College Mathematics Journal 33 (2002) 55; 34 (2003) $71-72$.

[4] Z. DARóCZY, Notwendige und hinreichende Bedingungen für die Existenz von nichtkonstanten Lösungen linearer Funktionalgleichungen, Acta Sci. Math. Szeged 22 (1961), 31-41.

[5] J. Dhombres, Some aspects of functional equations, Department of Mathematics, Chulalongkorn University, Bangkok, 1979.

[6] L. FuCHS, Infinite abelian groups, Vol. I, Academic Press, New York-San Francisco-London, 1970.

[7] I. Kaplansky, Infinite abelian groups, revised edition, University of Michigan Press, Ann Arbor, 1971.

[8] A. G. Kurosh, The theory of groups, Chelsea Publishing Company, New York, 1955.

[9] L. LosonczI, Bestimmung aller nichtkonstanten Lösungen von linearen Funktionalgleichungen, Acta Sci. Math. Szeged 25 (1964), 250-254.

[10] O. ORe, Structures and group theory II, Duke Math. J. 4 (1938), 247-269.

[11] H. PRÜFER, Untersuchungen über die Zerlegbarkeit der abzählbaren primären abelschen Gruppen, Math. Z. 17 (1923), 35-61.

[12] J. RÄтz, Characterizing projections of abelian groups. Report of the 40th International Symposium on Functional Equations, Aequationes Math. 65 (2003), 300.

[13] J. RÄтZ, Characterizing projections of abelian groups. II. Report of the 41st International Symposium on Functional Equations, Aequationes Math. 67 (2004), 298-299.

[14] A. Rosenfeld, An introduction to algebraic structures, Holden-Day, San FranciscoCambridge-London-Amsterdam, 1968.

\section{Jürg Rätz}

Mathematisches Institut

Universität Bern

Sidlerstrasse 5

CH-3012 Bern

Switzerland

Manuscript received: August 9, 2004 and, in final form, May 28, 2005. 